\title{
APPLICATION OF MULTI-FACETED FUNCTION MODEL BASED ON VONDRAK FILTER OPTIMIZATION IN UAV AERIAL IMAGE HEIGHT CORRECTION
}

\author{
Fei Zhou ${ }^{1}$, Lun $\mathrm{Pu}^{2 *}$, Shihua Tang ${ }^{2,3}$, Yifei Yang ${ }^{1}$ \\ ${ }^{1}$ Geomatices Center of Guangxi, No.5 Jianzheng Road, Nanning, Guangxi, 530023, China - gxzfei@sina.com \\ ${ }^{2}$ College of Geomatics and Geoinformation, Guilin University of Technology, No.12 Jian'gan Road, Guilin, Guangxi, \\ 541004,China-pulun16@163.com \\ ${ }^{3}$ Guangxi Key Laboratory of Spatial Information and Geomatics, No.12 Jian'gan Road, Guilin, Guangxi, 541004,China- \\ 58650875@qq.com
}

Commission IV, WG IV/3

KEY WORDS: Aerial image, Height correction, Vondrak filtering, Multi-Surface Function, Surface fitting, Precision analysis

\begin{abstract}
:
With the rapid development of drone technology and digital camera technology, the method of obtaining high-precision coordinates based on UAV aerial photogrammetry technology is popular. The plane coordinate accuracy of the aerial image of the drone has been able to meet the needs of practical applications, but the elevation accuracy is generally low. Aiming at the low elevation accuracy of UAV aerial photogrammetry, a multi-face function fitting method based on Vondrak filter optimization was proposed. The improved fitting model was used to obtain the elevation correction value of the aerial image, thereby obtaining high-precision image elevation data. In this paper, based on the traditional multi-face function fitting method, some known points were used to model and find the difference between the measured elevation value and the measured elevation. The Vondrak filter was used to smooth the fitting result. Finally, a small number of known elevation points were used for checking, so that the obtained elevation was compared with the actual elevation. The experimental comparison showed that the improved multi-face function fitting method used Vondrak filter was improved by $34.76 \%$ compared with the quadric surface fitting, and improved by $14.48 \%$ compared with the optimized cubic surface fitting method. Research shows that the multi-faceted function method based on Vondrak filtering is superior to the traditional elevation correction method. The experiment verifies the effectiveness and feasibility of the improved method, and provides some reference value for the research of aerial image elevation correction model.
\end{abstract}

\section{INTRODUCTION}

With the rapid development of UAV aerial photogrammetry technology, based on its flexible activities, fast response, high mapping accuracy and rich products, the UAV aerial photogrammetry is widely used in surveying and mapping (Zhang, X. X.,2019). Due to the influence of terrain fluctuations, camera attitude and hardware equipment during the mapping process, the measured elevation accuracy is generally low (Lin Y., 2019). In order to solve the problem of low elevation accuracy of UAV aerial photogrammetry products, many scholars have carried out a large number of experimental studies on the calculation method of elevation correction values (Wang Y., 2018). The elevation correction value fitting model is a common method. Due to the influence of observation data and terrain fluctuations, each fitting method has its limitations, it is difficult to meet the accuracy requirements required for actual production (Wang, Y. C.,2019)(Yin, Y. J., 2017). An optimal non-negative variable weight combination model based on plane fitting, quadric surface fitting and GA-BP neural network was proposed and the experimental comparison showed that the accuracy was improved (Li, F. D., 2017). Vondrank filtering can effectively smooth the observed data, so that the fitting results approach to the actual value, while multi-faceted function has a better fitting effect for the research area with complex topography, and can approach the actual research area by superposition of simple curved surface into any curved surface (Wu, Y. Y., 2012). In order to obtain more reasonable elevation innovation, this paper puts forward a multifaceted function fitting method based on Vondrank filtering improvement, and use filtering to process observed elevation innovation data and eliminate the influence of noise on the process of establishing the model, it provides more reasonable and effective reference basis for modelling to provide more reasonable reference basis for constructing the fitting model.

\section{VONDRAK FILTERING}

\subsection{Basic Vondrak Filter Description}

Vondrak filtering is a smoothing method based on the Whittaker smoothing method of the Czech astronomer J.Vondrak in the 1960s (Wu, Y. Y., 2011). The smoothness in the original definition is changed to the 3rd-order difference squared sum of the smoothed values. It was suitable for processing equally spaced or non-equidistant observations. The basic idea can be described by the following basic criteria:

$$
Q=F+\lambda^{2} S=\min
$$

In the above formula: $F$ is the fitting degree of the observation value, which reflects the approximation degree of the fitting curve and the observation curve; $S$ is the smoothness, and the smoothness of the curve is determined by the magnitude of the smoothed value.

About ten years later, J.Vondrak replaced the fitness and smoothness with their average values respectively in 1976. The improved basic criteria expressions are as follows:

\footnotetext{
* Corresponding author: Lun Pu, Email: pulun16@163.com
} 


$$
\begin{aligned}
& Q=F+\lambda^{2} S=\frac{1}{n} \sum_{i=1}^{n} p_{i}\left(y_{i}-y_{i}^{\prime}\right)^{2}+ \\
& \frac{\lambda^{2}}{n-3} \sum_{i=1}^{n-3}\left[\Delta^{3} y_{i}^{\prime}\right]^{2}=\min
\end{aligned}
$$

In equation (2): $n$ is the total number of observations; $p$ express the weight of the observation; $y_{i}$ is the observation, and $y_{i}^{\prime}$ is the smoothed value.

Defined the $\varepsilon=1 / \lambda^{2}$ as a smoothing factor, which balances the fitted and smoothed values (Li, H. R., 2012). The principle of Vondrak filter smoothing is to represent any adjacent four smoothed values by a cubic Lagrangian polynomial. The calculation rule of this polynomial is only applicable to the middle two points of the smoothed value. The $\Delta^{3} y_{i}^{\prime}$ express third-order difference representing the smoothed value, which is calculated as follows:

$$
\Delta^{3} y_{i}^{\prime}=a_{i} y_{i}^{\prime}+b_{i} y_{i+1}^{\prime}+c_{i} y_{i+2}^{\prime}+d_{i} y_{i+3}^{\prime}
$$

In the above formula, the coefficients $a, b, c$, and $d$ are calculated as follows:

$$
\begin{aligned}
& a_{i}=\frac{6 \sqrt{\left(x_{i+2}-x_{i+1}\right)(n-3) /\left(x_{n-1}-x_{2}\right)}}{\left(x_{i}-x_{i+1}\right)\left(x_{i}-x_{i+2}\right)\left(x_{i}-x_{i+3}\right)} ; \\
& b_{i}=\frac{6 \sqrt{\left(x_{i+2}-x_{i+1}\right)(n-3) /\left(x_{n-1}-x_{2}\right)}}{\left(x_{i+1}-x_{i}\right)\left(x_{i+1}-x_{i+2}\right)\left(x_{i+1}-x_{i+3}\right)} ; \\
& c_{i}=\frac{6 \sqrt{\left(x_{i+2}-x_{i+1}\right)(n-3) /\left(x_{n-1}-x_{2}\right)}}{\left(x_{i+2}-x_{i}\right)\left(x_{i+2}-x_{i+1}\right)\left(x_{i+2}-x_{i+3}\right)} ; \\
& d_{i}=\frac{6 \sqrt{\left(x_{i+2}-x_{i+1}\right)(n-3) /\left(x_{n-1}-x_{2}\right)}}{\left(x_{i+3}-x_{i}\right)\left(x_{i+3}-x_{i+1}\right)\left(x_{i+3}-x_{i+2}\right)} .
\end{aligned}
$$

According to the expression (1), in order to minimize the value of $Q$, the condition is satisfied: $\frac{\partial Q}{\partial y^{\prime}}=\frac{\partial F}{\partial y^{\prime}}+\lambda^{2} \frac{\partial S}{\partial y^{\prime}}=0$, suppose $\lambda \neq 0$, Get the basic equations of the Vondrak filter:

$$
\sum_{k=-3}^{3} A_{k, i} y_{k+i}^{\prime}=B_{i} \quad i=1,2, \cdots, n
$$

Among them, the coefficients are as follows:

$$
\begin{aligned}
& A_{-3, i}=a_{i-1} d_{i-3} ; \\
& A_{-2, i}=a_{i-2} c_{i-2}+b_{i-3} d_{i-3} ; \\
& A_{-1, i}=a_{i-1} b_{i-1}+b_{i-2} c_{i-2}+c_{i-3} d_{i-3} ; \\
& A_{0, i}=a_{i}^{2}+b_{i-1}^{2}+c_{i-2}^{2}+d_{i-3}^{2}+\varepsilon p_{i} /(n-3) ; \\
& A_{1, i}=a_{i} b_{i}+b_{i-1} c_{i-1}+c_{i-2} d_{i-2} ; \\
& A_{2, i}=a_{i} c_{i}+b_{i-1} d_{i-1} ; A_{3, i}=a_{i} d_{i}
\end{aligned}
$$

Combined with the above relationship, the Vondrak filtering result of the observed values can be obtained by solving the solution of the equation (4).

\subsection{Weighted Least Squares Vondrak Filter}

Considering the fact that the observed values may contain gross errors, in order to avoid the influence of gross error on the modeling, the weight matrix was introduced to the basic Vondrak filtering method, and the filtering method was optimized by weighted least squares to make it resistant to gross error. Combined with the basic criteria, the Vondrak filtered least squares improved expression is described as follows:

$$
\boldsymbol{Q}=\boldsymbol{V}^{\mathrm{T}} \boldsymbol{P} \boldsymbol{V}=\min
$$

In equation (5), the specific calculation expressions of each parameter are as follows:

$$
\begin{aligned}
& \boldsymbol{V}=\left[\begin{array}{l}
\frac{1}{\sqrt{n}} \boldsymbol{E}_{n \times n} \\
\frac{\lambda}{\sqrt{n-3}} \boldsymbol{A}_{(n-3) \times n}
\end{array}\right] \hat{\boldsymbol{Y}}-\left[\begin{array}{l}
\frac{1}{\sqrt{n}} \boldsymbol{Y}_{n \times 1} \\
\mathbf{0}_{(n-3) \times n}
\end{array}\right] \quad \hat{\boldsymbol{Y}}=\left[\begin{array}{c}
\hat{y}_{1} \\
\hat{y}_{2} \\
\vdots \\
\hat{y}_{n}
\end{array}\right] \\
& \boldsymbol{Y}=\left[\begin{array}{c}
y_{1} \\
y_{2} \\
\vdots \\
y_{n}
\end{array}\right] \\
& \boldsymbol{P}=\left[\begin{array}{ll}
\boldsymbol{D}_{n \times n} & \\
& \boldsymbol{E}_{(n-3) \times(n-3)}
\end{array}\right]
\end{aligned}
$$

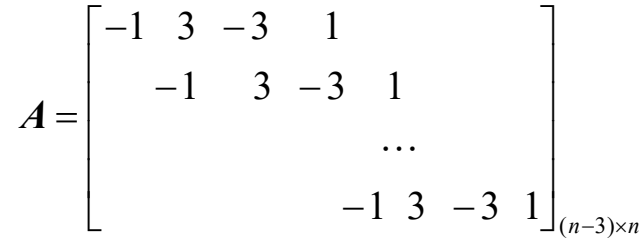

Among them, the matrix $\boldsymbol{A}$ is a strip-type diagonal matrix, which is only related to the parameter n, and the matrix $\boldsymbol{E}$ is an identity matrix. The matrix $\boldsymbol{D}$ is the product of the weight matrix and the correction matrix, namely:

$$
\boldsymbol{D}=\left[\begin{array}{cccc}
p_{1} & & & \\
& p_{2} & & \\
& & \ddots & \\
& & & p_{n}
\end{array}\right]\left[\begin{array}{cccc}
\rho_{1} & & & \\
& \rho_{2} & & \\
& & \ddots & \\
& & & \rho_{n}
\end{array}\right]
$$

After the derivative calculation and simplification, the following expression is obtained:

$$
\hat{\boldsymbol{Y}}=\left(\boldsymbol{D}+\frac{n}{n-3} \lambda^{2} \boldsymbol{A}^{\mathrm{T}} \boldsymbol{A}\right)^{-1} \boldsymbol{D} \boldsymbol{Y}
$$

The Vondrak filter with improved weighted least squares is more convenient and concise. According to equation (6), the filtered sequence value can be obtained by simply setting the appropriate smoothing factor.

\section{MULTI-SURFACE FUNCTION}

The multifaceted function is a process of approximating an arbitrary rounded surface with a number of single-valued mathematical faces (Tian X., 2017). The specific method of fitting is to create a mathematical surface at each data point, superimposing the rotating surfaces in a certain proportion in each direction and forming a continuous curved surface to pass the fitted data points. According to the multi-face function fitting principle, let the known surface function be $f(x, y)$. If the function $\varphi(x, y)$ satisfies the equation follows:

$$
\sum\left(f\left(x_{i}, y_{i}\right)-\varphi\left(x_{i}, y_{i}\right)\right)^{2}=\min
$$

We call $\varphi(x, y)$ is surface function of the function $f(x, y)$. The approximation function $\varphi(x, y)$ is expressed as the following expression: 


$$
\varphi(x, y)=\sum_{j=1}^{u} \beta_{j} Q_{j}\left((x, y),\left(x_{j}, y_{j}\right)\right)
$$

In the above formula: $\beta$ is the undetermined coefficient, $Q$ represents the kernel function, $u$ indicates the number of kernel functions, $\left(x_{j}, y_{j}\right)$ is the node coordinates of the selected central position.

We can choose any simple function as the kernel function, but in order to make the calculation more convenient, we usually choose the symmetric or distance function as the kernel function. The fitting of spatial data can be divided into "clock" type kernel function and "bowl" type kernel function. The following three kinds of kernel functions are often used in multi-face function fitting:

Positive hyperbolic function expression:

$$
Q=\left(\left(x-y_{j}\right)^{2}+\left(y-y_{j}\right)^{2}+\delta\right)^{1 / 2}
$$

Inverted hyperbolic function expression:

$$
Q=\left(\left(x-y_{j}\right)^{2}+\left(y-y_{j}\right)^{2}+\delta\right)^{-1 / 2}
$$

Cubic surface function expression:

$$
Q=\left(\left(x-y_{j}\right)^{2}+\left(y-y_{j}\right)^{2}\right)^{3 / 2}+\delta
$$

In the above formula, $\delta$ represents the kernel function smoothing factor, which can be used to adjust the shape of the kernel function.

With $n$ sets of observations $\left(x_{i}, y_{i}, \zeta_{i}\right), i=1,2, \cdots$, According to the above formula, the error equation is obtained as follows:

$$
\begin{aligned}
& v_{1}=\sum_{j=1}^{u} \hat{\beta}_{j} Q_{j}\left(\left(x_{1}, y_{1}\right),\left(x_{0 j}, y_{0 j}\right)\right)-\zeta_{1} \\
& v_{2}=\sum_{j=1}^{u} \hat{\beta}_{j} Q_{j}\left(\left(x_{2}, y_{2}\right),\left(x_{0 j}, y_{0 j}\right)\right)-\zeta_{2} \\
& \ldots \\
& v_{n}=\sum_{j=1}^{u} \hat{\beta}_{j} Q_{j}\left(\left(x_{n}, y_{n}\right),\left(x_{0 j}, y_{0 j}\right)\right)-\zeta_{n}
\end{aligned}
$$

In the formula (12), $\left(x_{0 j}, y_{0 j}\right)$ represents the coordinates of the central node, and $\zeta$ is the observation corresponding to the central coordinate, and the matrix is expressed as:

$$
V=A \hat{\beta}-\zeta
$$

In the above formula, each matrix is represented as follows:

$$
\begin{aligned}
V & =\left(v_{1}, v_{2}, \cdots, v_{n}\right)^{T} \\
\hat{\beta} & =\left(\hat{\beta}_{1}, \hat{\beta}_{2}, \cdots, \hat{\beta}_{u}\right)^{T} \\
\zeta & =\left(\zeta_{1}, \zeta_{2}, \cdots, \zeta_{n}\right)^{T} \\
\underset{n \times u}{A} & =\left(\begin{array}{l}
Q_{1}\left(\left(x_{1}, y_{1}\right),\left(x_{01}, y_{01}\right)\right), \cdots, Q_{u}\left(\left(x_{1}, y_{1}\right),\left(x_{0 u}, y_{0 u}\right)\right) \\
Q_{1}\left(\left(x_{2}, y_{2}\right),\left(x_{01}, y_{01}\right)\right), \cdots, Q_{u}\left(\left(x_{2}, y_{2}\right),\left(x_{0 u}, y_{0 u}\right)\right) \\
\cdots \\
Q_{1}\left(\left(x_{n}, y_{n}\right),\left(x_{01}, y_{01}\right)\right), \cdots, Q_{u}\left(\left(x_{n}, y_{n}\right),\left(x_{0 u}, y_{0 u}\right)\right)
\end{array}\right)
\end{aligned}
$$

According to equation (13), using the principle of least squares, the coefficient terms can be obtained as follows:

$$
\hat{\beta}=\left(A^{T} A\right)^{-1} A^{T} \zeta
$$

After solving the coefficient matrix $\beta$ and substituting into equation(8), the multi-face function fitting model can be obtained, and then the function value of the to-be-determined point can be obtained according to the known coordinates.

\section{POLYNOMIAL SURFACE FITTING}

The basic idea of surface function fitting model is based on the functional relationship between the plane coordinates of the known points in the measurement area and the corresponding elevation correction values, the smooth surface in the measurement area is fitted by the numerical function, and the actual elevation value of the fixed points is obtained. There are quadric surface fitting method and cubic surface fitting method in common polynomial surface fitting (Xiong, Z. Q., 2017).

The elevation correction of the observation point has the following relations with the plane coordinates:

$$
\xi=f(x, y)+v
$$

We call $f(x, y)$ is surface function of the function $\xi . v$ is the fit residual. Expanding the function $\mathrm{f}$ in the above equation according to the polynomial can be expressed as:

$$
\begin{aligned}
& f(x, y)=a_{0}+a_{1} x+a_{2} y+a_{3} x^{2}+a_{4} y^{2}+ \\
& a_{5} x y+a_{6} x^{3}+a_{7} y^{3}+a_{8} x^{2} y+a_{9} x y^{2}+\cdots
\end{aligned}
$$

According to the above two expressions, the error equation can be written into matrix form as follows:

$$
\boldsymbol{V}=\boldsymbol{B} \boldsymbol{X}-\boldsymbol{\zeta}
$$

In the error equation (17), each matrix is represented as:

$$
\begin{gathered}
\boldsymbol{B}=\left[\begin{array}{ccccccc}
1 & x_{1} & y_{1} & x_{1}^{2} & y_{1}^{2} & x_{1} y_{1} & \cdots \\
1 & x_{2} & y_{2} & x_{2}^{2} & y_{2}^{2} & x_{2} y_{2} & \cdots \\
& \vdots & \\
1 & x_{n} & y_{n} & x_{n}^{2} & y_{n}^{2} & x_{n} y_{n} & \cdots
\end{array}\right] \\
\boldsymbol{V}=\left[\begin{array}{c}
v_{1} \\
v_{2} \\
\vdots \\
v_{n}
\end{array}\right] \quad \boldsymbol{X}=\left[\begin{array}{c}
a_{1} \\
a_{2} \\
\vdots \\
a_{n}
\end{array}\right] \quad \boldsymbol{\zeta}=\left[\begin{array}{c}
\xi_{1} \\
\xi_{2} \\
\vdots \\
\xi_{n}
\end{array}\right]
\end{gathered}
$$

According to the least squares method, the solution in (17) can be obtained as:

$$
\boldsymbol{X}=\left(\boldsymbol{B}^{\mathrm{T}} \boldsymbol{B}\right)^{-1} \boldsymbol{B}^{\mathrm{T}} \boldsymbol{\zeta}
$$

The quadric surface fitting model takes the first six terms in equation (16). There are 6 coefficients in the model. The cubic surface fitting method selects the first 10 terms in equation (16), that is, the 10 coefficients of the demand solution model. The coefficient term $\mathrm{X}$ in the formula (18) is substituted into the equation (16) to obtain the elevation correction value of the point to be determined.

\section{THE CORRECTION EXPERIMENT AND THE PRECISION ANALYSIS}

The experimental data is derived from the literature $(\mathrm{Lu}, \mathrm{X}$. P., 2014), the point distribution is not uniform, the first 9 points are selected as the known data to establish the elevation correction model, and the data of the last 9 points is used to check the fitting accuracy of the model. The difference between the actual elevation of the point and the measured elevation is taken as the known data of the fitting. At the same time, the plane 
coordinates of each control point are considered, and the plane coordinates are normalized and used for building a model experiments.

The same batch of data is processed by quadratic surface fitting method and multi-face function fitting method respectively. The elevation correction number was calculated when Vondrank filtering was not added, and then using Vondrank filtering to improve the fitting residuals in different cases shown in Table 1.

\begin{tabular}{cccccccccccc}
\hline \multirow{2}{*}{$\begin{array}{c}\text { Point } \\
\text { number }\end{array}$} & $\begin{array}{c}\text { Known } \\
\text { data }(m)\end{array}$ & $\begin{array}{c}\text { No filter } \\
\text { fitting } \\
\text { result }(m)\end{array}$ & $\begin{array}{c}\text { Residual } \\
(m)\end{array}$ & $\begin{array}{c}\text { Filtered } \\
\text { fitting } \\
\text { result }(m)\end{array}$ & $\begin{array}{c}\text { Residual } \\
(m)\end{array}$ & & $\begin{array}{c}\text { No filter } \\
\text { fitting } \\
\text { result }(m)\end{array}$ & $\begin{array}{c}\text { Residual } \\
(m)\end{array}$ & $\begin{array}{c}\text { Filtered } \\
\text { fitting } \\
\text { result }(m)\end{array}$ & $\begin{array}{c}\text { Residual } \\
(m)\end{array}$ \\
\hline 10 & -0.092 & -0.2162 & -0.1242 & -0.3092 & -0.2172 & & -0.2514 & -0.1594 & -0.2090 & -0.1170 \\
11 & -0.546 & -0.3085 & 0.2375 & -0.2108 & 0.3352 & & -0.3395 & 0.2065 & -0.2465 & 0.2995 \\
12 & -0.232 & -0.4139 & -0.1819 & -0.2134 & 0.0186 & & -0.3391 & -0.1071 & -0.2703 & -0.0383 \\
13 & -0.143 & -0.1648 & -0.0218 & -0.2426 & -0.0996 & & -0.2550 & -0.1120 & -0.2227 & -0.0797 \\
14 & -0.159 & -0.4219 & -0.2629 & -0.2873 & -0.1283 & & -0.5037 & -0.3447 & -0.3135 & -0.1545 \\
15 & -0.642 & -0.2001 & 0.4419 & -0.2631 & 0.3789 & & -0.1298 & 0.5122 & -0.2662 & 0.3758 \\
16 & -0.196 & -0.2838 & -0.0878 & -0.3062 & -0.1102 & & -0.2889 & -0.0929 & -0.2945 & -0.0985 \\
17 & -0.164 & -0.3145 & -0.1505 & -0.2111 & -0.0471 & & -0.3231 & -0.1591 & -0.2169 & -0.0529 \\
18 & -0.088 & -0.6599 & -0.5719 & -0.1782 & -0.0902 & & -0.4858 & -0.3978 & -0.2317 & -0.1437 \\
\hline
\end{tabular}

Table 1. Using Vondrak filter to improve the previous fitting method and improved fitting residual comparison results

It can be seen from Table 1. that whether it is a quadric surface fitting or a multi-faceted function fitting, the residual difference obtained by adding Vondrank filtering is much smaller than the residual value improved without filtering, and the overall fitting is the residual is more stable.

Through calculation and comparison, the accuracy of the quadric surface calculated by the improved method is $\pm 0.2842 \mathrm{~m}$, and the improved fitting accuracy by using Vondrank filtering is $\pm 0.1979 \mathrm{~m}$, and the improved fitting accuracy is improved by about $30 \%$. In the multi-face function fitting method, the accuracy of not using filtering is $\pm 0.2719 \mathrm{~m}$, and the improved fitting result by adding Vondrank filtering is $\pm 0.1854 \mathrm{~m}$, and the overall accuracy is improved by about $31.8 \%$. It can be seen that the improved method of fitting the elevation correction value is superior to the traditional method, and the accuracy of the fitting model is greatly improved.

In order to further illustrate the effectiveness of the improved method, the improved multi-face function fitting method is compared with the quadric and cubic surface fitting results. The comparison of the multi-face function fitting method improved by Vondrank filtering with the uncorrected fitting method of the quadric surface and the residual of the improved cubic surface fitting method are shown in Table 2. At the same time, the accuracy of the three methods is calculated separately and a reasonable evaluation is made.

\begin{tabular}{|c|c|c|c|c|c|c|}
\hline \multirow[b]{2}{*}{ Point number } & \multicolumn{2}{|c|}{ Quadric surface fitting } & \multicolumn{2}{|c|}{ Cubic surface fitting } & \multicolumn{2}{|c|}{ Multi-faceted function fitting } \\
\hline & $\begin{array}{l}\text { Unimproved } \\
\operatorname{method}(m)\end{array}$ & $\operatorname{Residual}(m)$ & $\begin{array}{c}\text { Filter } \\
\text { optimized }(m)\end{array}$ & $\operatorname{Residual}(m)$ & $\begin{array}{c}\text { Vondrak } \\
\text { improvement }(m)\end{array}$ & $\operatorname{Residual}(m)$ \\
\hline 10 & -0.2162 & -0.1242 & -0.3755 & -0.2835 & -0.2090 & -0.1170 \\
\hline 11 & -0.3085 & 0.2375 & -0.2457 & 0.3003 & -0.2465 & 0.2995 \\
\hline 12 & -0.4139 & -0.1819 & -0.2346 & -0.0026 & -0.2703 & -0.0383 \\
\hline 13 & -0.1648 & -0.0218 & -0.2925 & -0.1495 & -0.2227 & -0.0797 \\
\hline 14 & -0.4219 & -0.2629 & -0.3668 & -0.2078 & -0.3135 & -0.1545 \\
\hline 15 & -0.2001 & 0.4419 & -0.3218 & 0.3202 & -0.2662 & 0.3758 \\
\hline 16 & -0.2838 & -0.0878 & -0.3473 & -0.1513 & -0.2945 & -0.0985 \\
\hline 17 & -0.3145 & -0.1505 & -0.2605 & -0.0965 & -0.2169 & -0.0529 \\
\hline 18 & -0.6599 & -0.5719 & -0.3163 & -0.2283 & -0.2317 & -0.1437 \\
\hline Precision & & \pm 0.2842 & & \pm 0.2168 & & \pm 0.1854 \\
\hline
\end{tabular}

Table 2. Compare the accuracy analysis results of the three fitting methods

According to the residual results calculated in Table 2. and the accuracy of the three methods, the accuracy of the fitting model improved by Vondrank filtering is the highest among the three fitting methods, that is, the accuracy of the checking is $\pm 0.1854 \mathrm{~m}$, which is better than the other two. The method has a good fitting effect. Although the improved cubic surface fitting method is better than the quadratic surface fitting method before the improvement, the overall improvement accuracy is not very large, so it is not the best fitting scheme. At the same time, it can be seen that by comparing the fitting residuals of the nine checkpoints, the improved method is more stable and the residual fluctuation range is smaller. The improved method is more suitable for the fitting calculation of the elevation correction value.

In order to verify the validity and feasibility of Vondrank filtering, the filtering improvement method is applied to quadratic surface and multi-faceted function respectively for fitting calculation. Firstly, Vondrank filtering is added to two different fitting methods, and the calculation method is added when no filtering is added. The residual results are obtained for comparison. The calculated four groups of fitted residuals are shown in Figure 1. 


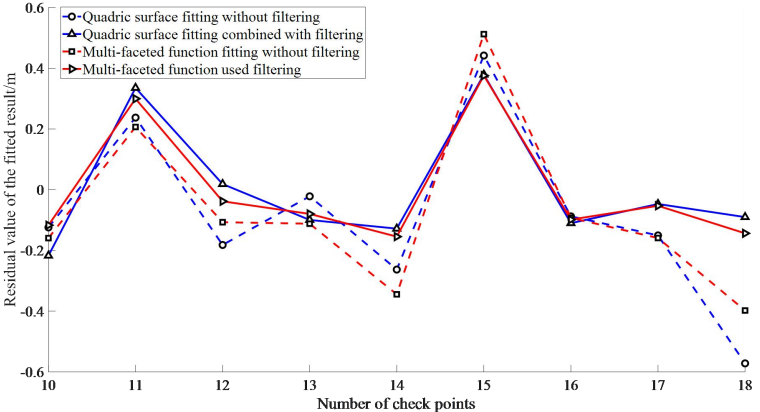

Figure 1. Check results of quadric surface and polyhedral function before and after used filtering

It can be seen from Fig.1 that the solid line graph is the improved fitting residual after adding Vondrank filtering, and the two solid lines are close to the place where the residual result is the smallest. Among them, the red solid line is the multi-faceted function plus the improved residual of Vondrank filter, which is also the most stable curve, which is much better than the unfiltered method. The solid blue line is the improved trend of quadratic surface fitting plus Vondrank filtering. This method is slightly lower than the improved multi-faceted function, but much more accurate than when no filtering is used. It can be seen that the improved fitting method using Vondrank filtering is more effective and feasible.

The above method was compared with the cubic surface fitting method improved by filtering, and the fitting residual trend chart shows in Figure 2.

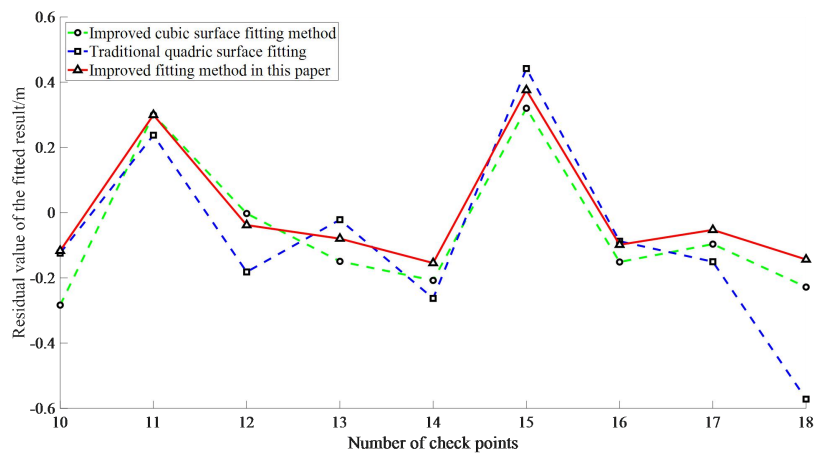

Figure 2. Results of residual error comparison between improved Vondrank filtering and traditional method

Figure 2. clearly shows the comparison of the three methods. The red solid line is the improved fitting method studied in this paper. The overall trend tends to be stable, and the fitting result is better than the other two methods.

\section{CONCLUSION}

Aiming at the problem of low elevation accuracy in UAV aerial photogrammetry technology, this paper used Vondrank filter to improve the method of fitting the elevation correction value of multi-faceted function. The improved multi-faceted function was compared with the traditional quadric and cubic surfaces respectively, and the superiority of the method after adding Vondrank filtering was verified. The comparative analysis shows that the improved method was $34.76 \%$ more accurate than the quadric surface fitting method, and $14.88 \%$ higher than the accuracy of the three-optimized surface fitting method. The experimental research shows that the improved multi-face function fitting method based on Vondrank filtering is effective and feasible in the aerial photography elevation correction of $\mathrm{UAV}$, and has obvious effect on improving the fitting precision.
It has certain reference value and guiding significance for actual production.

\section{ACKNOWLEDGEMENTS}

This paper is financially supported by GuangXi Natural Science Foundation under grant numbers 2018GXNSFAA138168, National Natural Science of China under Grant numbers 41864002, and GuangXi Key Laboratory of Spatial Information and Geomatics Program under Grant numbers 16-380-25-13.

\section{REFERENCES}

Zhang, X. X., Wang, S. T., Li, Y. C., et al, 2019: Accuracy analysis of aerial small UAV flight control attitude data. Science of Surveying and Mapping 44(5),103-109.

Lin Y., Li, X. T., Wu, S. Y., et al, 2019. Application of tilt photogrammetry with fixed-wing UAV better than $2 \mathrm{~cm}$ resolution in topographic survey. Science of Surveying and Mapping(6),http://kns.cnki.net/kcms/detail/11.4415.p.20190624. 0831.005.html.

Wang Y., Peng, Q. S., Tan, R. C., et al, 2018, Airborne LiDAR submarine point cloud filtering method based on moving surface fitting. Geospatial Information 16(6), 21-25.

Wang, Y. C., Yu, J. Y, Tian, M. Y., et al, 2019. High accuracy elevation correction method for vehicle-borne moving surveying system. Remote Sensing Information 34(2), 86-90.

Yin, Y. J., Zhang, Z. Y., Gao, D. J., 2017, Study of Elevation Correction Method of Inshore Underwater Points. Journal of Geomatics 42(01), 34-37.

Li, F. D., Tang, S. H., Liang, Y. J., 2017: Optimal non-negative variable weight combination for elevation correction of aerial images. Remote Sensing Information 32(6), 122-126.

Wu, Y. Y., Zhu, J. J., Zuo, T. Y., 2012: The Application of Vondrak Filtering Combined with multisurface Function in height Anomaly Fitting. Geotechnical Investigation \& Surveying (7), 58-61.

Wu, Y. Y., Zhu, J. J., Zuo, T. Y., 2011: Application of 2-D Vondrak Filtering in Height Abnormal Fitting. Journal of Geodesy and Geodynamics 31(6), 132-135.

Li, H. R., Yang, Z. Q., Si Z., et al, 2012, Application of Vondrak Filter in Data Processing of Magnetic Suspension Gyro-total-station. Journal of Earth Sciences and Environment 34(4), 107-110

Tian X., Zheng, H. Y., Zhang C., et al, 2017, Optimization Selection of kernel function in fitting region vertical deformation field using multi-surface function. North China Earthquake Sciences 35(4), 11-15.

Xiong, Z. Q., Xiao, T. F., 2017: Realization of GPS Height Quadratic Surface Fitting Using MATLAB and C Language. Innovation Science and Technology (17), 57.

Lu, X. P., 2014. Empirical study on the mapping precision based on UAV low-altitude photogrammetry. China University of Mining and Technology, Xuzhou, 44-50. 